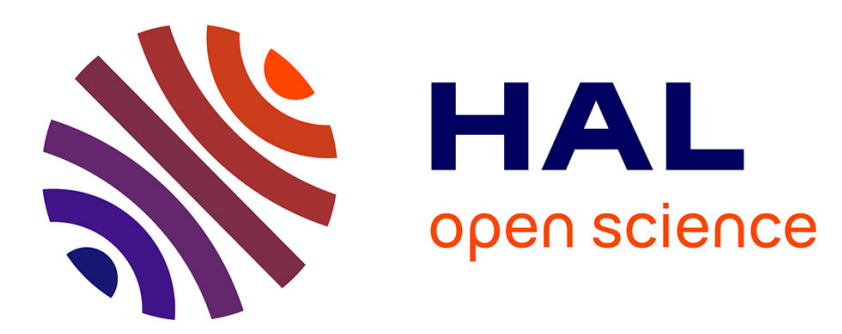

\title{
Delay modeling of high-speed distributed interconnect for the signal integrity prediction
}

\author{
B. Raveloa
}

\section{To cite this version:}

B. Raveloa. Delay modeling of high-speed distributed interconnect for the signal integrity prediction. European Physical Journal: Applied Physics, 2012, 57 (3), 10.1051/epjap/2012110374 . hal00788685

\section{HAL Id: hal-00788685 \\ https://hal.science/hal-00788685}

Submitted on 15 Feb 2013

HAL is a multi-disciplinary open access archive for the deposit and dissemination of scientific research documents, whether they are published or not. The documents may come from teaching and research institutions in France or abroad, or from public or private research centers.
L'archive ouverte pluridisciplinaire HAL, est destinée au dépôt et à la diffusion de documents scientifiques de niveau recherche, publiés ou non, émanant des établissements d'enseignement et de recherche français ou étrangers, des laboratoires publics ou privés. 


\title{
Delay modelling of high-speed distributed interconnect for the signal integrity prediction
}

\author{
Blaise Ravelo \\ IRSEEM (Research Institute in Electronic and Embedded Systems), EA 4353, \\ Graduate school of engineering ESIGELEC, \\ Technopole du Madrillet, Avenue Galilée, BP 10024, \\ 76801 Saint Etienne du Rouvray Cedex, France. \\ Tel: +33.(0)2.32.91.59.71 / Fax: +33.(0)2.32.91.59.71. \\ E-mail: blaise.ravelo@yahoo.fr
}

\begin{abstract}
A relevant modelling method of distributed interconnect line for the high-speed signal integrity (SI) application is introduced in this paper. By using the microwave and transmission line (TL) theory, the interconnect lines are assumed as its distributed RLC-model. Then, based-on the transfer matrix analysis, the second order global transfer function of the interconnect network comprised of the TL driven by voltage source including its internal resistance and the impedance load is expressed. Thus, mathematical analysis enabling the physical SI parameters extraction was established by using the transient response of the loaded line. To verify the relevance of the developed model, RC- and RLC-lines excited by square-wave-pulse with 10-Gbits/s-rate were investigated. So, comparisons with SPICE-computations were performed. As results, transient responses perfectly well-correlated to the reference SPICE-models were evidenced. As application of the introduced model, evaluations of rise-/fall- times, propagation delays, signal attenuations and even the settling times were realized for different values of TL parameters. Compared to other methods, the computation execution time and data-memory consumed by the program implementing the proposed delay modelling-method algorithm are much better.
\end{abstract}

Keywords: Delay modelling, high-speed interconnects, mixed signal processing, RC-/RLC-line, signal integrity.

\section{INTRODUCTION}

In the late of 1950s, thanks to the deed scientific and physical analyses on the semi-conductor materials, the mankind discovers the era of microelectronics. This industrial period was born with the invention of the integrated circuits (ICs) by Jack S. Kilby from Texas Instruments [1]. The impact of this invention was awarded with the Nobel Prize in physical discipline in 2000. In the middle of the 1960s, the Intel co-founder Gordon Moore who is one of the pioneers in Silicon Valley [2-3], formulated an empirical law stating that the performance of ICs, including the number of components on it, doubles every 18-24 months with the same chip price. With this spectacular progress, the understanding of the physical effect in the components is fundamental. Then, one of the most developed mathematical prediction enabling to foresee the progress of the microelectronic 
circuits performance is known as Moore's law was proposed [4].

Till now, the electronic technology develops with higher integration scale according to this analytical prediction which manifests with the increase of the operating frequency and the integration density [5-7]. For the better understanding in the matter of the basic functioning of this technology, a large scientific background on the physical approach permitting an accurate analysis of the circuit and system equivalent behaviours or electrical modelling, electromagnetic (EM) effects on the wave propagation and also the mathematical approach for the signal theory are necessary [8-13]. The synergy of all these multiphysic fields constitutes the particularity of the signal integrity (SI) discipline which is one of major steps for investigating the analogue and digital high-speed systems.

Despite the spectacular technological progress of microelectronic systems, the complexity of the IC structures including the increase of their interconnect density, which link several million of logic gates, becomes more and more sophisticated. Beyond the unintentional perturbation phenomena as the electromagnetic interference (EMI) and electromagnetic compatibility (EMC) emissions [8-9], this electronic printed circuit board (PCB) and IC design complexity can generate systematically a serious problematic in terms of SI and power integrity (PI) of the interconnection networks [10-13].

To cope with this issue, the design research engineers must innovate their techniques by taking into account the electrical interconnection influences. Usually, the mathematical predictions enabling to model the undesired physical aspects (loss, distorsion, delay, overshoot...) induced by the interconnect structures are based on the electrical model defined by the per-unit length parameters RLCG [14-16]. Till now, the most popular theory used for the analytical investigations of the interconnect structure are based on the Elmore [17] and Wyatt [1820] models named also as lumped RC-model are used by most industrial semi-conductor designers for estimating the typical linear system transient responses. In fact, the models introduced and used in [17-20] are fundamentally developed with the first order approximation of the interconnect system operating-voltage transfer function. Despite this simplicity, since the late 1990s, it has been underlined that with such an approximation, compared to second order lumped models [21-28], the transient response parameters as the rise-/fall- times and the propagation delay can present relative errors more than 30-\%. Furthermore, the first order model is not sufficient for analyzing certain phenomena as the current or voltage under- and over-shoots. For this reason, with the current electronic systems operating frequency, which achieves some $\mathrm{GHz}$, the second order parameters as the inductance effect become more and more significant in the high-speed interconnect networks as PCBs, ICs packages and even connectors. One of the simplest existing delay models recently proposed [23][25][28-30] is based on the linear systems with canonical transfer function written as:

$$
T(s)=\frac{T_{0}}{s^{2}+2 \zeta \cdot \omega_{a} \cdot s+\omega_{a}^{2}},
$$

with $T_{0}$ is a real constant, and $\omega_{a}$ and $\zeta$ are respectively the undamped natural angular frequency and damping ratio. But this model is valid only for the transfer function with linear polynomial forms. So, further and deeper analysis is necessary for the complex systems governed by typically non-linear transfer functions as the distributed transmission lines (TL). 
To do this, an example of technique for compensating the degradation induced by interconnect lines is introduced in [24-25][28][31]. More recently, interconnect effects equalization technique, particularly, for reducing the signal delays [32-33] based on the use of the negative group delay circuit whose the principle is developed in [34-35] has been introduced. Moreover, accurate, optimized and also easy to implement models enabling the prediction of these unwanted effects are indispensable [36-39]. In this scope, new generations of design strategies and commercial numerical tools for simulation and characterization of various 3-D structure geometries permitting to ensure the signal fidelity at Gbits/s-speeds were recently reported [40-44]. But, the computed results with these tools remain critical when the designed structure like entire systems (SiP, SoC, PoP, BGA packages...) present many levels of integration. Nowadays, easier and more relevant modelling method of electronic circuit interconnect network is still needed.

For this reason, a modelling-method based on the distributed interconnection network is developed in this paper. For the clearance of readability, this paper is structured in three sections. Section 2 describes the fundamental approach enabling to determine the transfer function of the distributed electronic network interconnection system. Then, basic matrix theory applied to the generalized periodical lumped circuit constituting the assumed interconnect system will be proposed. In addition, a mathematical analysis of the established model unit-step response enabling to the calculation of the SI-parameters as the propagation delay, rise-/fall-times, settling time and even the attenuation will be carried out. Hence, validations through computations with the reference tool SPICE-environment will be offered in Section 3. Lastly, Section 4 is the conclusion and some prospects of this work.

\section{DESCRIPTION OF THE ANALOG HIGH-SPEED INTERCONNECT SYSTEM CONSIDERED}

Fig. 1 depicts the considered interconnect system comprised of a transmission line (TL) having characteristic impedance $Z_{c}$ and physical length $d$ driven by a voltage source $v_{i}(t)$ having internal impedance $R_{s}$ and loaded by an impedance $R_{L} C_{L}$-parallel. The voltage across the output load of this circuit is denoted $v_{o}(t)$.

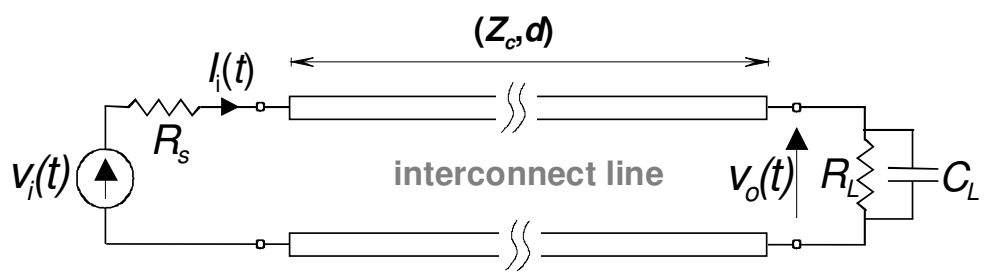

Fig. 1: Interconnect line with characteristic impedance, $Z_{c}$ and physical length, $d$ driven by a voltage source, $v_{i}(t)$ with internal impedance $R_{S}$ loaded by $R_{L} C_{L}$-parallel network.

In order to investigate the integrity of the mixed or analogue-digital signals propagating through the whole interconnection system shown in Fig. 1, a relevant knowledge about the analytical behaviour of equivalent transfer function is essentially indispensable. In fact, this allows the extraction of different transient or timedomain parameters. For that one can proceed with a theoretical approach based on the exploitation of the equivalent transfer matrix of each circuit block constituting the whole system. 


\subsection{Analysis of distributed line model}

By using the interconnect input impedance expressed in appendix (A-4) and according to Ohm's law, the input current $I_{i}(s)$ injected in the circuit shown in Fig. 1 should be written as:

$$
I_{i}(s)=\frac{V_{i}(s)}{R_{s}+Z_{i n}(s)}=\frac{V_{i}(s)}{R_{s}+Z_{c} \frac{Z_{L} \cosh (\gamma \cdot d)+Z_{c} \sinh (\gamma \cdot d)}{Z_{L} \sinh (\gamma \cdot d)+Z_{c} \cosh (\gamma \cdot d)}} .
$$

In order to achieve more explicit analytical development, the transfer function $T(s)$ must be expressed in function of the basic Laplace variable $s$. This leads us naturally to the examination of the transfer function regarding the distributed TL model with the per-unit length parameters $R, L$ and $C$.

According to the microwave- and TL- theories, by taking into account the implicit resistive metallic loss, and the inductive and capacitive effects of the interconnect line, the lossy-line introduced in Fig 1 can be assumed as a distributed RLC-network model schematized in Fig. 2. The parameters $R_{u}, L_{u}$ and $C_{u}$ represent respectively, the per-unit length resistance, inductance and capacitance of the interconnect line and $\delta x$ is an infinitesimal small physical length.

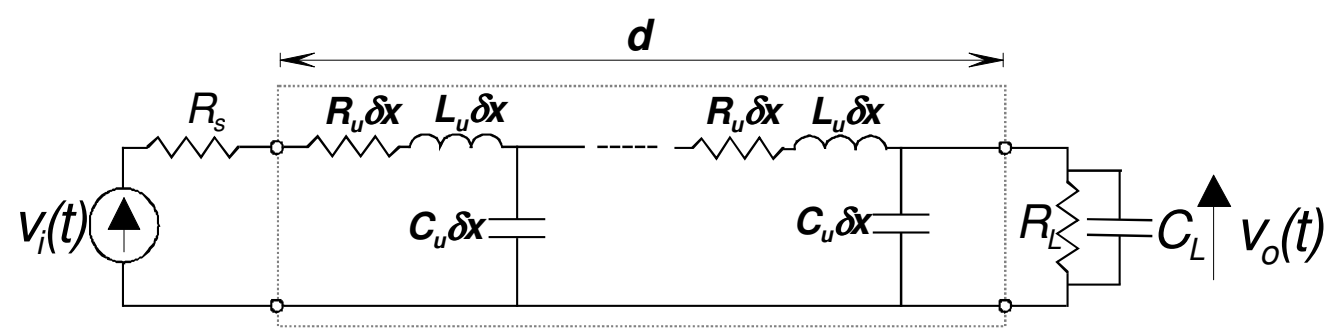

Fig. 2: Distributed circuit equivalent of the RLC-model of the system shown in Fig. 1.

In this case, the transfer function expressed in formula (A-3) is transformed as follows:

$$
\begin{aligned}
T(s)= & \frac{d \sqrt{\left(R_{u}+L_{u} \cdot s\right) \cdot C_{u} \cdot s} \cdot R_{L}}{d \sqrt{\left(R_{u}+L_{u} \cdot s\right) \cdot C_{u} \cdot s}\left[R_{L}+R_{s}\left(1+R_{L} \cdot C \cdot s\right)\right] \cosh \left(d \sqrt{\left(R_{u}+L_{u} \cdot s\right) \cdot C_{u} \cdot s}\right)} \\
& +\left[d^{2}\left(R_{u}+L_{u} \cdot s\right) \cdot C_{u} \cdot s\left(1+R_{L} \cdot C \cdot s\right)+R_{s} R_{L}\right] \sinh \left(d \sqrt{\left(R_{u}+L_{u} \cdot s\right) \cdot C_{u} \cdot s}\right)
\end{aligned}
$$

By replacing $s$ by $j \omega$, due to the presence of the terms $\cosh ($.$) and \sinh ($.$) , the transmittance of the structure$ under study can be determined. From there, we can deduce the time-domain response through fast Fourier transform (fft). But the accuracy of the fft and inverse fft (ifft) remains critical when the bandwidth of the input signal is larger than $10 \mathrm{GHz}$. In addition, the exploitation of the frequency response to deduce the transient characteristics of the transfer system (as overshoot, rise-time, time-response) is impossible. For this reason, a polynomial approach permitting the extractions of these transient parameters is developed in the next section. 


\subsection{Extraction of the transient response parameters}

In fact, the assessment of the SI-parameters suited to the distributed TL understudy from this non-polynomial transfer function is mathematically very complicated because the transient response is relatively complicated to express analytically. For this reason, one proposes to exploit the distributed RLC-network described in Fig. 2. Thanks to the MacLaurin polynomial expanding of the denominator, the transfer function in (A-3) can be approximated as:

$$
T(s)=\frac{1}{c_{0}+c_{1} \cdot s+c_{2} \cdot s^{2}+O\left(s^{3}\right)},
$$

with

$$
\begin{gathered}
c_{0}=1+\frac{R_{s}+R_{u} \cdot d}{R_{L}}, \\
c_{1}=\left(\frac{R_{u} \cdot C_{u}}{2}+\frac{6 L_{u}+3 R_{s} \cdot C_{u} \cdot R_{u}+R_{u}^{2} \cdot C_{u} \cdot d}{6 R_{L}}\right) d^{2}+\left(R_{s} \cdot C_{u}+R_{u} \cdot C_{L}\right) d, \\
c_{2}=\left(\frac{R_{u}^{2} \cdot C_{u} \cdot d^{2}}{24}+\frac{L_{u}}{2}+\frac{R_{u} \cdot L_{u} \cdot d}{3 R_{L}}+\frac{R_{u}^{3} \cdot C_{u} \cdot d^{3}}{120 R_{L}}\right) \cdot C_{u} \cdot d^{2} \\
+\left[L_{u} \cdot C_{L}+R_{s} \cdot d\left(\frac{R_{u} \cdot C_{u}^{2} \cdot d}{6}+\frac{R_{u} \cdot C_{u} \cdot C_{L}}{2}+\frac{L_{u} \cdot C_{u}}{2 R_{L}}+\frac{R_{u}^{2} \cdot C_{u}^{2} \cdot d^{2}}{24 R_{L}}\right)\right] d
\end{gathered}
$$

By identification between the second-order approximation of transfer function expressed in (4) and the canonical form introduced earlier in (1), one can write the following parameters:

$$
\begin{aligned}
& T_{0}=\frac{1}{c_{2}}, \\
& \omega_{a}=\sqrt{\frac{c_{0}}{c_{2}}}, \\
& \zeta=\frac{c_{1}}{2 \sqrt{c_{0} \cdot c_{2}}} .
\end{aligned}
$$

According to the damping ratio value $\zeta$, one distinguishes three different cases of unit-step responses which enable to determine the transient response parameters prior to the evaluation of the output SI-parameters.

It is interesting to note that the limit of the model presented here depends on two parameters. First, by denoting $f_{\max }$, the maximal operating frequency and $v$, the speed of the signal propagating along the interconnect line, the rise-time of the input signal which must be higher than [15]: 


$$
t_{r} \geq \frac{0.35}{f_{\max }}=\frac{2.8 d}{v}
$$

Then, according to the limited polynomial expansion, the inaccuracies of the model can be evaluated with the high-order terms of the transfer function expressed in (3). For example, the influence of the dominant third-term can be considered with the series MacLaurin expansion by the third-order coefficient expressed as follows for the RLC-interconnect model:

$$
c_{3}=\frac{C_{u} d^{2}}{24}\left[R_{s} C_{L} R_{u}^{2} C_{u} d^{2}+2 L_{u}\left(2 R_{s} C_{u} d+6 R_{s} C_{L}+4 R_{u} C_{L} d+R_{u} C_{u} d^{2}+\frac{R_{s} R_{u} C_{u} d^{2}+2 L_{u} d}{R_{L}}\right)\right] .
$$

In difference with the model established in [45], here, the root of the equation $v_{o}(t)=0$ with the direct calculation enabling achieving more accurate and precise mathematical expressions is considered. So, in the remainder of this section, the detailed formulae on the different SI parameters are offered.

\subsubsection{Case $1, \zeta<1$}

It is well-known that in this case, the under consideration system behaves as a classical overdamped system. Clearly, it should present an overshoot here denoted by $\xi$ at the time, $T_{\xi}$ which are respectively, expressed as:

$$
\begin{gathered}
\xi=e^{\frac{-\pi \zeta}{\sqrt{1-\zeta^{2}}}} \text { if } \zeta<1, \\
T_{\xi}=\frac{\pi}{\omega_{a} \sqrt{1-\zeta^{2}}} .
\end{gathered}
$$

Hence, the $\pm 5-\%$ settling time is defined as:

$$
t_{s \pm 5 \%} \approx \frac{3}{\zeta \cdot \omega_{a}}
$$

More importantly, the $50 \%$ propagation delay is estimated as:

$$
T_{p} \approx \frac{0.5 T_{\xi}}{1+e^{\frac{-\pi \zeta}{\sqrt{1-\zeta^{2}}}}}
$$

In the present case, by approximating the output voltage $v_{o}(t)$ as its tangential line passing through its unique inflection point, one demonstrates the here below rise-time formulation: 


$$
t_{r} \approx 0.8 \frac{\omega_{a}}{T_{0}} e^{\frac{\zeta}{\sqrt{1-\zeta^{2}}} \arctan \left(\sqrt{\zeta^{-2}-1}\right)} .
$$

\subsubsection{Case 2, $\zeta=1$}

In this case, the normalized output unit step response is literally written as:

$$
v_{o}(t)=1-\left(1+\omega_{a} \cdot t\right) e^{-\omega_{a} \cdot t} .
$$

For the better understanding, one denotes $t=T_{\lambda}$ the root of the equation $v_{o}(t)=\lambda$ ( $\lambda$ is real constant positive lower than 1). With this expression, this parameter can be extracted easily by considering a singular mathematical function. The corresponding solution can be expressed as:

$$
T_{\lambda}=\frac{L_{W}\left[e^{-1}\left(\frac{\lambda \cdot \omega_{a}^{2}}{T_{0}}-1\right)\right]+1}{\omega_{a}},
$$

where $L_{W}(x)$ expresses the mathematical Lambert function of $x$, which is defined as a mathematical function satisfying the following equation [46-47]:

$$
L_{W}(x) \cdot e^{L_{W}(x)}=x
$$

Nevertheless, simpler formulations of the 50-\% propagation delay and the rise-time are estimated as:

$$
\begin{aligned}
& T_{p} \approx \frac{1}{\omega_{a}}, \\
& t_{r} \approx \frac{\ln (9) \sqrt{2}}{\omega_{a}} .
\end{aligned}
$$

\subsubsection{Case 3, $\zeta>1$}

In this case, it is obvious that $T(s)$ behaves as an underdamped system with two real poles:

$$
\begin{gathered}
\omega_{1}=\frac{1}{\tau_{1}}=\omega_{a}\left(\zeta+\sqrt{\zeta^{2}-1}\right) \\
\omega_{2}=\frac{1}{\tau_{2}}=\omega_{a}\left(\zeta-\sqrt{\zeta^{2}-1}\right)
\end{gathered}
$$


Moreover, by exploiting the dominant term (here based on the contribution of the pole $\omega_{2}$ ) of the normalized unit step response for high value of the temporal parameter $t$, the $\pm 5-\%$ settling time is established as:

$$
t_{s \pm 5 \%} \approx \tau_{2}\left[3+\ln \left(\tau_{2}\right)-\ln \left(\tau_{2}-\tau_{1}\right)\right]
$$

Similar to case 1 , by approximating the output $v_{o}(t)$ as its tangential line passing through its unique inflection point, the propagation delay demonstrated is written as follows:

$$
T_{p} \approx \frac{\left[\lambda_{2}+1\right] \tau_{2}-\left[\lambda_{1}+1\right] \tau_{1}}{\lambda_{2}-\lambda_{1}}+\frac{0.5\left(\omega_{1}-\omega_{2}\right)}{T_{0}\left(\lambda_{2}-\lambda_{1}\right)}+\frac{\ln \left(\frac{\omega_{2}}{\omega_{1}}\right)}{\omega_{2}-\omega_{1}}
$$

with

$$
\begin{aligned}
& \lambda_{1}=\left(\frac{\omega_{2}}{\omega_{1}}\right)^{\frac{\omega_{1}}{\omega_{1}-\omega_{2}}}, \\
& \lambda_{2}=\left(\frac{\omega_{2}}{\omega_{1}}\right)^{\frac{\omega_{2}}{\omega_{1}-\omega_{2}}} .
\end{aligned}
$$

Finally, knowing that in this case of underdamped system, the whole transfer function is formed by the product of transfer function having rise-times $\ln (9) \tau_{1}$ and $\ln (9) \tau_{2}$, the overall rise time can be estimated as:

$$
t_{r} \approx \ln (9) \sqrt{\tau_{1}^{2}+\tau_{2}^{2}}
$$

In general way, regarding the three cases cited previously, it is clear that the normalized unit step response voltage attenuation $\alpha\left(t_{0}\right)$ at the given instant time $t=t_{0}$ is explicitly defined as:

$$
\alpha\left(t_{0}\right)=\left\{\begin{array}{c}
\frac{T_{0}}{\omega_{a}^{2}}\left[1-\left(1+\omega_{a} t_{0}\right) e^{-\omega_{a} t_{0}}\right] \text { if } \zeta=1 \\
\frac{T_{0}}{\omega_{a}^{2}}\left\{1-e^{-\omega_{a} t_{0}}\left[\cosh \left[\omega_{a} t_{0} \sqrt{\zeta^{2}-1}\right]+\frac{\zeta}{\sqrt{\zeta^{2}-1}} \sinh \left[\omega_{a} t_{0} \sqrt{\zeta^{2}-1}\right]\right]\right\} \text { if } \zeta>1 . \\
\frac{T_{0}}{\omega_{a}^{2}}\left\{1-\frac{e^{-\zeta \omega_{a} t_{0}}}{\sqrt{1-\zeta^{2}}} \sin \left[\omega_{a} t_{0} \sqrt{1-\zeta^{2}}+\arctan \left(\frac{\sqrt{1-\zeta^{2}}}{\zeta}\right)\right]\right\} \text { if } \zeta<1
\end{array}\right.
$$

To get further insight about the efficiency of this theoretical concept, validation toward SPICE-computation environment of the ADS-software from Agilent ${ }^{\mathrm{TM}}$ is performed in the next section. 


\section{APPLICATION}

This section focus is on the comparisons of time-domain results computed from the proposed modelling method and those directly simulated with SPICE. It is interesting to note that the Spice which uses mainly computation technique based on the nodal calculation method is the industry standard for electrical simulation because of its accuracy and the availability of free source code.

\subsection{Numerical experiments with distributed high-speed RC-line (particular case of RLC-line with $L_{u}=0$ )}

Fig. 3 depicts the design schematic of the simulated interconnection network. It consists of the distributed RCmodel available in ADS library with per-unit length resistance $R_{u}=5 \Omega / \mathrm{mm}$ and capacitance $C_{u}=0.5 \mathrm{pF} / \mathrm{mm}$, and physical length $d=5 \mathrm{~mm}$, which is loaded by $R_{L}=100 \Omega$ and $C_{L}=\{0.1 \mathrm{pF}, 1 \mathrm{pF}, 2 \mathrm{pF}\}$. This interconnection circuit is excited by a normalized square waveform pulse voltage with amplitude $V_{M}=1 \mathrm{~V}$ having internal impedance $R_{s}=5 \Omega$ and time duration $t_{0}=100$ ps. It corresponds as well to a digital data-source with $10 \mathrm{Gbit} / \mathrm{s}-$ rate.

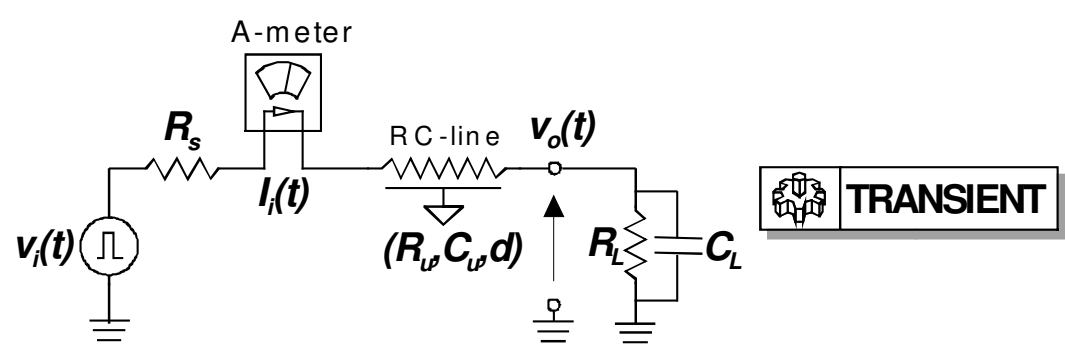

Fig. 3: ADS-schematic of the tested circuit comprised of $\mathrm{RC}$-line driven by square-wave source having 10Gbits/s-rate and loaded by $R_{L} C_{L}$-network.

After transient simulation run from $t_{\min }=0$ to $t_{\max }=300 \mathrm{ps}$ and step $\Delta t=0.1 \mathrm{ps}$, one gets the comparative results displayed in Fig. 4(a) for the output voltage and shown in Fig. 4(b) for the time-dependent input current regarding the normalized unit step input.

The transient response from the reference SPICE-computation regarding the distributed RC-line model is plotted in grey full line and those computed from the proposed model is plotted in black dashed line. So, one observes a very good agreement between the transient responses from the ADS-model and the proposed model responses. One evaluates here relative errors which are mainly due to the numerical inaccuracy of lower than 5-\%. In the case where $R_{s}$ and $C_{L}$ negligible and by varying the per-unit length resistance $R_{u}=\{5 \Omega / \mathrm{mm}, 10 \Omega / \mathrm{mm}, 15$ $\Omega / \mathrm{mm}$ \} of the circuit shown in Fig. 3 through ADS sweep simulation, one gets the transient results displayed in Fig. 5 with the normalized unit step input voltage. We can remark that as predicted in theory, the 50-\% propagation delay and the output attenuation increase when $R_{u}$ increases. 


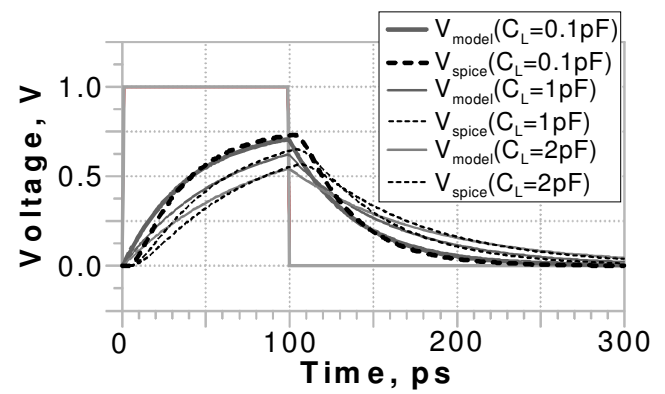

(a)

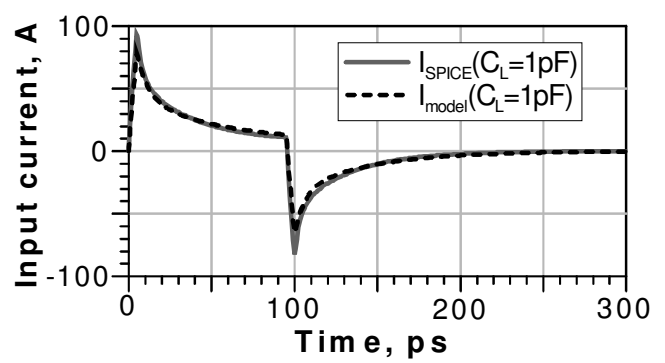

(b)

Fig. 4: Transient result comparisons from ADS and from the model proposed

$$
\left(R_{u}=5 \Omega / \mathrm{mm}, C_{u}=0.5 \mathrm{pF} / \mathrm{mm}, d=5 \mathrm{~mm} \text { and } R_{L}=100 \Omega\right) \text {. }
$$

Furthermore, to check the effectiveness of the mathematical analysis on the transient response parameters detailed in subsection 2.2, comparisons with SPICE-computations were realized. Therefore, the results summarized in Table 1 are obtained. It shows the assessments of the proposed different transient parameters as the propagation delay, rise-/fall-times and also the voltage attenuation expressed in (30) at the given instant time $t_{0}$.

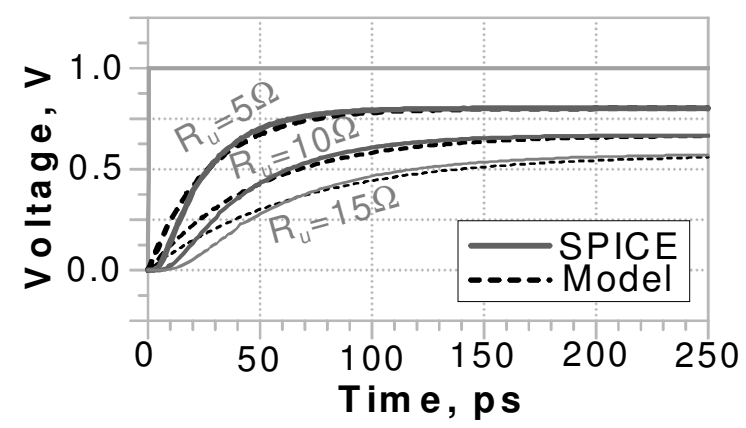

Fig. 5: Unit step responses for $R_{u}=\{5 \Omega / \mathrm{mm}, 10 \Omega / \mathrm{mm}, 15 \Omega / \mathrm{mm}\}$, $C_{u}=0.5 \mathrm{pF} / \mathrm{mm}, d=5 \mathrm{~mm}$ and $R_{L}=100 \Omega$.

One points out that the results presented were calculated by implementing the formulation established with the scientist standard tool Matlab. The attenuation can be estimated as the final value when the given pulse duration $t_{0}$ is higher than the settling time. To do so, via formulae (4) and (5), by taking $s=0$, the steady state final values $T(0)=\{0.80,0.67,0.57\}$ accordingly to $R_{u}=\{5 \Omega / \mathrm{mm}, 10 \Omega / \mathrm{mm}, 15 \Omega / \mathrm{mm}\}$ were found. 


\begin{tabular}{l|l|l}
\hline \hline$R_{u}$ & SPICE & Proposed model \\
\hline \multirow{3}{*}{$5 \Omega / \mathrm{mm}$} & $T_{p}=30 \mathrm{ps}$ & $T_{p} \approx 27 \mathrm{ps}$ \\
& $t_{r}=49 \mathrm{ps}$ & $t_{r} \approx 51 \mathrm{ps}$ \\
& $\alpha\left(t_{0}\right)=0.79$ & $\alpha\left(t_{0}\right) \approx 0.76$ \\
\hline \multirow{3}{*}{$10 \Omega / \mathrm{mm}$} & $T_{p}=64 \mathrm{ps}$ & $T_{p} \approx 59 \mathrm{ps}$ \\
& $t_{r}=86 \mathrm{ps}$ & $t_{r} \approx 89 \mathrm{ps}$ \\
& $\alpha\left(t_{0}\right)=0.60$ & $\alpha\left(t_{0}\right) \approx 0.54$ \\
\hline \multirow{3}{*}{$15 \Omega / \mathrm{mm}$} & $T_{p}=119 \mathrm{ps}$ & $T_{p} \approx 101 \mathrm{ps}$ \\
& $t_{r}=113 \mathrm{ps}$ & $t_{r} \approx 120 \mathrm{ps}$ \\
& $\alpha\left(t_{0}\right)=0.45$ & $\alpha\left(t_{0}\right) \approx 0.40$ \\
\hline \hline
\end{tabular}

Table 1: Comparison between the SI parameter calculated with the proposed model and SPICEcomputations.

As the settling time for $R_{u}=5 \Omega / \mathrm{mm}$ is of about $t_{s} \approx 76 \mathrm{ps}$ then lower than the input pulse duration $t_{0}=100 \mathrm{ps,}$ the attenuation $\alpha\left(t_{0}\right)$ can be considered as equal to $T(0)$. As confirmed by Table 1 , the results from the proposed model are much closed to the SPICE-computation. It means that to preserve the output voltage amplitude as higher as possible, one should operate with digital signal having pulse duration absolutely higher than the TL network settling time $t_{s}$. In the next section, the investigation of the RLC-model by taking into account the inductive parameter of the TL is presented.

\subsection{Numerical experiments with distributed high-speed RLC-line}

With the same configuration as in the previous subsection, an RLC-model interconnection network as presented earlier in Fig. 2 were designed and simulated. So, the results depicted in Fig. 6 are realized. Similar to the former study, these simulations were performed with the equivalent distributed network comprised of the RLC-model having per-unit length resistance $R_{u}=5 \Omega / \mathrm{mm}$ with various sweep inductance values $L_{u}=\{0.2 \mathrm{nH} / \mathrm{mm}, 0.4$ $\mathrm{nH} / \mathrm{mm}, 0.6 \mathrm{nH} / \mathrm{mm}\}$ and capacitance $C_{u}=0.5 \mathrm{pF} / \mathrm{mm}$ and with physical length $d=5 \mathrm{~mm}$. The RLC-line tested is driven by a voltage source delivering a unit step signal having amplitude $V_{M}=1 \mathrm{~V}$ and loaded by $R_{L}=100 \Omega$. After transient simulation run from $t_{\min }=0$ to $t_{\max }=500 \mathrm{ps}$ step $\Delta t=2 \mathrm{ps}$, the time domain computation results model are displayed in Fig. 6. In difference with the case of RC-lines, here, overshoot phenomena and significant pure delays are occurred. It is underlined that the inductive effects increase with the length of interconnects. Despite these effects, we can find that once again, as forecasted in theory, the signal 50-\% propagation delay increases with the per-unit inductance $L_{u}$. 


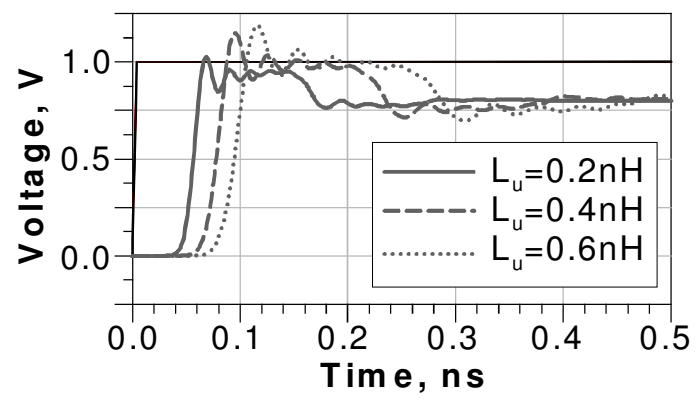

Fig. 6: Transient unit step simulation results from the proposed RLC-model for $R_{u}=5 \Omega / \mathrm{mm}$, various sweep inductances $L_{u}=\{0.2 \mathrm{nH} / \mathrm{mm}, 0.4 \mathrm{nH} / \mathrm{mm}, 0.6 \mathrm{nH} / \mathrm{mm}\}$ and capacitance $C_{u}=0.5 \mathrm{pF} / \mathrm{mm}$ with physical length $d=5 \mathrm{~mm}$ loaded by $R_{L}=100 \Omega$.

In addition, according to the comparative results with respect to the calculation of the propagation delay parameter, the numerical values addressed in Table 2 for different values of per-unit length inductance $L_{u}$ are realized. Compared with the results presented in [45], we emphasize that for the interconnect line with 5-mm length, better values of propagation delay relative errors are confirmed.

\begin{tabular}{c|c|c}
\hline \hline$L_{u}$ & SPICE & Proposed model \\
\hline $0.2 \mathrm{nH} / \mathrm{mm}$ & $T_{p}=57 \mathrm{ps}$ & $T_{p} \approx 56 \mathrm{ps}$ \\
\hline $0.4 \mathrm{nH} / \mathrm{mm}$ & $T_{p}=78 \mathrm{ps}$ & $T_{p} \approx 75 \mathrm{ps}$ \\
\hline $0.6 \mathrm{nH} / \mathrm{mm}$ & $T_{p}=95 \mathrm{ps}$ & $T_{p} \approx 89 \mathrm{ps}$ \\
\hline \hline
\end{tabular}

Table 2: Comparison of the RLC-line propagation delays calculated with the proposed model and SIPCEcomputations.

To sum up the all above investigations, concluding remarks is drawn in the next section.

\section{CONCLUDING REMARKS}

A modelling-method of distributed interconnection network for the high-speed SI-analysis was investigated. The developed model is based the interconnect TL comprised of RLC-network. For that the TL is essentially characterized by its per-unit electrical parameters $\mathrm{R}, \mathrm{L}$ and $\mathrm{C}$ and its physical length.

Thanks to the exploitation of the under consideration system transfer matrix, the second-order analytical approximation of the global transfer function was established. Then, it was demonstrated mathematically how to determine accurately the transient response parameters such as rise-time, 50-\% propagation delay and settling time. It was found that the developed model provides an easy way for the SI-parameter evaluations whose the exact and usually non-linear interconnect-system transfer function remains analytically very complicated to explore. In order to evidence the relevance of the introduced theoretic formulations yielded from the developed model, simulations of circuitry comprised of loaded RC- and RLC-lines were carried out. Then, comparisons with SPICE-computations were also performed by considering input square-wave pulse voltage having 10- 
Gbits/s-rate in function of the TL-parameters.

The main benefits of the presented modelling-method lie on its simplicity, its flexibility for the integration in the computation tools for the prediction of the layout trace as the PCB or typical chip-to-chip interconnection. Furthermore, the following remarks were found out:

1) The proposed modelling-method enables to extract efficiently transient parameters.

2) This generates attenuation permitting to predict the level of the output voltage according to the input signal time duration.

3) The programming implementing the investigated method algorithm executed with Matlab was run during very less computation time only in order of $\mathrm{ms}$ for generating considerably accurate results.

4) The developed discrete model can be adapted to the computed-aided design of all mixed or analogue-digital electronic system dedicated in particular to the SI characterization.

In the next step of this work, the application of the validated modelling-method for the prediction of the high frequency analogue-digital module operating at several Gbits/s is outlined. In addition, the perturbation SI effects as reflections, ringing and crosstalk will be investigated by considering the total transfer matrices of systems comprised of multi-conductor interconnect lines.

\section{APPENDIX}

According to the circuit and system theory, the transfer matrix of the TL characterized by its transfer impedance $Z_{c}$, propagation constant $\gamma$ and geometrical length $d$ is defined as:

$$
\left[M_{T L}\right]=\left[\begin{array}{cc}
\cosh (\gamma \cdot d) & Z_{c} \sinh (\gamma \cdot d) \\
\frac{\sinh (\gamma \cdot d)}{Z_{c}} & \cosh (\gamma \cdot d)
\end{array}\right]
$$

where $\gamma$ is the propagation constant of the under consideration TL. So that, it is well-known that the global transfer matrix of the overall system (TL driven by a source having internal resistance $R_{s}$ cascaded with a shunt resistance $R_{L}$ ) introduced above in Fig. 1 is equal to the following matrix product:

$$
[M(s)]=\left[\begin{array}{cc}
1 & R_{s} \\
0 & 1
\end{array}\right] \cdot\left[\begin{array}{cc}
\cosh (\gamma \cdot d) & Z_{c} \sinh (\gamma \cdot d) \\
\frac{\sinh (\gamma \cdot d)}{Z_{c}} & \cosh (\gamma \cdot d)
\end{array}\right] \cdot\left[\begin{array}{cc}
1 & 0 \\
\frac{1}{R_{L}}+C_{L} \cdot s & 1
\end{array}\right]
$$

As argued previously, to treat the SI propagating through this system, one should realize theoretic analysis based on the accurate analytical expression of the transfer function. By definition, the transfer function (here denoted $T(s))$ associated to the above transfer matrix $[M(s)]$ is merely given by the inverse of the first element $M_{11}(s)$ : 


$$
\begin{aligned}
T(s)=\frac{V_{o}(s)}{V_{i}(s)}=\frac{1}{M_{11}(s)}= & \frac{Z_{c} \cdot R_{L}}{Z_{c}\left[R_{L}+R_{s}\left(1+R_{L} \cdot C \cdot s\right)\right] \cosh (\gamma \cdot d)} . \\
& +\left[Z_{c}^{2}\left(1+R_{L} \cdot C \cdot s\right)+R_{s} R_{L}\right] \sinh (\gamma \cdot d)
\end{aligned}
$$

By denoting $Z_{L}(s)=R_{L}+1 / C . s$, the input impedance $Z_{i n}(s)$ of the loaded TL can be deduced literally from the transfer matrix elements $M_{11}(s)$ and $M_{21}(s)$ via the following expression:

$$
Z_{i n}(s)=\frac{M_{11}(s)}{M_{21}(s)}=Z_{c} \frac{Z_{L}(s) \cosh (\gamma \cdot d)+Z_{c} \sinh (\gamma \cdot d)}{Z_{L}(s) \sinh (\gamma \cdot d)+Z_{c} \cosh (\gamma \cdot d)}
$$

With regard to the RLC-model shown in Fig. 2, one recalls that the characteristic impedance and the propagation constant are respectively, theoretically defined as follows:

$$
\begin{gathered}
Z_{c}=\sqrt{\frac{R_{u}+L_{u} \cdot s}{C_{u} \cdot s}}, \\
\gamma=\sqrt{\left(R_{u}+L_{u} \cdot s\right) \cdot C_{u} \cdot s} .
\end{gathered}
$$

\section{REFERENCES}

[1] J. S. Kilby, "Invention of the integrated circuits," IEEE Trans. on Electron Devices, Vol. 23, p. 648, July 1976.

[2] G. E. Moore, "Cramming More components onto integrated circuits", Electronics Magazine, Vol. 38, No. 8, pp. 114-117, Apr. 1965.

[3] G. E. Moore, "Progress in digital integrated electronics," Proceedings of the IEEE International Electron Devices Meeting, pp. 11-13, Dec. 1975.

[4] Moore's Law. [Online]. Available: http://www.intel.com/museum/archives/historydocs/mooreslaw.htm D.K. Sharma, B.K. Kaushik and R.K. Sharma, "VLSI interconnects and their testing: prospects and challenges ahead", Journal of Engineering, Design and Technology, Vol. 9, No. 1, pp. 63 - 84, 2011.

[5] N. Magen, A. Kolodny, U. Weiser and N. Shamir, "Interconnect-power dissipation in a microprocessor," Proceedings of the ACM International Workshop on System Level Interconnect Prediction, pp. 7-13, Feb. 2004.

[6] X.-C. Li Jun-Fa Mao Hui-Fen Huang Ye Liu, "Global interconnect width and spacing optimization for latency, bandwidth and power dissipation," IEEE Transactions on Electron Devices, Vol. 52, No. 10, pp. 2272-2279, Oct. 2005.

[7] M. A. Sayed and E. Y. A. Maksoud, "Interconnect synthesis in high speed digital VLSI routing", Int. J. Open Problems Compt. Math., Vol. 2, No. 3, pp. 383-415, Sep. 2009.

[8] M. Voutilainen, M. Rouvala, P. Kotiranta and T. Rauner, "Multi-Gigabit serial link emissions and mobile terminal antenna interference," in Proc. of 13th IEEE Workshop on SPI, Strasbourg, France, May 2009. 
[9] T. Sato, Y. Cao, K. Agarwal, D. Sylvester and C. Hu, "Bidirectional closed-form transformation between on-chip coupling noise waveforms and interconnect delay-change curves”, IEEE Trans. on Computer-Aided Design of Integrated Circuits and Systems, Vol. 22, No. 5, pp. 560-572, May 2003.

[10] S. Hasan, A-K. Palit and W. Anheier, "Equivalent victim model of the coupled interconnects for simulating crosstalk induced glitches and delays," in Proc. of 13th IEEE Workshop on SPI, Strasbourg, France, May 2009.

[11] W. Maichen, "When digital becomes analog-interfaces in high speed test," Tutorial course, in Proc. of 12th IEEE Workshop on SPI, Avignon, France, May 2008.

[12] A. C. Scogna, A. Orlandi and V. Ricciuti, "Signal and power integrity performances of striplines in presence of 2D EBG planes," in Proc. of 12th IEEE Workshop on SPI, Avignon, France, May 2008.

[13] V. Champac, V. Avendano and J. Figueras, "Built-in sensor for signal integrity faults in digital interconnect signals," IEEE Tran. VLSI, Vol. 18, No. 2, pp. 256-269, Feb. 2010.

[14]T. Eudes, B. Ravelo, and A. Louis, "Transient response characterization of the high-speed interconnection RLCG-model for the signal integrity analysis,” PIER Journal, Vol. 112, pp. 183-197, 2011.

[15] B. Ravelo and T. Eudes, "Fast estimation of RL-loaded microelectronic interconnections delay for the signal integrity prediction," To be published in Int. J. of Numerical Modelling: Electronic Networks, Devices and Fields (Int. J. Numer. Model). DOI: 10.1002/jnm.838

[16] T. Eudes, B. Ravelo, and A. Louis, "Experimental validations of a simple PCB interconnect model for highrate signal integrity", To be published in IEEE Trans. EMC, DOI: 10.1109/TEMC.2011.2165216.

[17] W. C. Elmore, "The transient response of damped linear networks," J. Appl. Phys., Vol. 19, pp. 55-63, Jan. 1948.

[18]L. Wyatt, "Circuit analysis, simulation and design. North-Holland," The Netherlands: Elsiever Science, 1978.

[19] Jr. J. L. Wyatt, “Waveform bounding for fast timing analysis of MOS VLSI circuits," in 1983 Proc. of IEEE ISCAS, pp. 760-761.

[20] Jr. J. L. Wyatt and Q. Yu, "Signal delay in RC meshes, trees and lines," in 1984 Proc. of IEEE ICAD, pp. 15-17.

[21] A. Deutsch, "High-speed signal propagation on lossy transmission lines," IBM J. Res. Develop., Vol. 34, No. 4, Jul. 1990, pp. 601-615.

[22]D. Deschacht, "Impact of inductance and routing orientation on timing performances of coupled interconnect lines”, 2010 International Conference on Design \& Technology of Integrated Systems in Nanoscale Era.

[23] A. B. Kahng, and S. Muddu, “An analytical delay model of RLC interconnects," IEEE Trans. CAD, Vol. 16, Dec. 1997, pp. 1507-1514.

[24]L. P. P. P. van Ginneken, "Buffer placement in distributed RC-tree network for minimal Elmore delay", Proceedings of the IEEE International Symposium of Circuits and Systems, pp. 865-868, May 1990.

[25] V. Adler and E. G. Friedman, "Repeater design to reduce delay and power in resistive interconnect," IEEE Trans. Circuits Syst. II, Analog and Digital Signal Processing, Vol. 54, No. 5, May 1998, pp. 607-616.

[26] A. Ligocka and W. Bandurski, "Effect of inductance on interconnect propagation delay in VLSI circuits," in Proc. of 8th Workshop on SPI 9-12 May 2004, Heidelberg, Germany, pp. 121-124. 
[27] A. K. Palit, V. Meyer, K. K. Duganapalli, W. Anheier and J. Schloeffel, "Test pattern generation based on predicted signal integrity loss through reduced order interconnect model," in Proc. of 16th Workshop Test Methods and Reliability of Circuits and Systems, Mar. 2004, pp. 84-88.

[28] V. Adler and E. G. Friedman, "Repeater Design to Reduce Delay and Power in Resistive Interconnect," IEEE Trans. Circuits Syst. II, Analog and Digital Signal Processing, Vol. 54, No. 5, May 1998, pp. 607616.

[29] Y. I. Ismail, E. G. Friedman and J. L. Neves, "Equivalent Elmore Delay for RLC Trees," IEEE Tran. CAD, Vol. 19, No. 1, Jan. 2000, pp. 83-97.

[30] Y. I. Ismail and E. G. Friedman, "Effects of inductance on the propagation, delay and repeater insertion in VLSI circuits,” IEEE Tran. VLSI, Vol. 8, No. 2, Apr. 2000, pp. 195-206.

[31]P. K. Hanumolu, G. Y. Wei and U. K. Moon, "Equalizers for high speed serial links," Int. J. High Speed Electronics and Systems, Vol. 15, No. 2, pp. 429-458, 2005.

[32]B. Ravelo, A. Perennec and M. Le Roy, "New technique of inter-chip interconnect effects equalization with negative group delay active circuits," INTECH Book, VLSI, Chap. 20, Edited by Z. Wang, Feb. 2010, pp. 409-434.

[33]T. Eudes and B. Ravelo, "Cancellation of delays in the high-rate interconnects with UWB NGD active cells”, Applied Physics Research, Vol. 3, No. 2, pp. 81-88, Nov. 2011.

[34] B. Ravelo, "Investigation on microwave negative group delay circuit", Electromagnetics, Vol. 31, No. 8, pp. 537-549, Nov. 2011.

[35] B. Ravelo, "Demonstration of negative signal delay with short-duration transient pulse", Eur. Phys. J. Appl. Phys. (EPJAP), Vol. 55, 10103, pp. 1-8, 2011.

[36] A. Nieuwoudt, J. Kawa and Y. Massoud, "Crosstalk-induced delay, noise, and interconnect planarization implications of fill metal in nanoscale process technology," IEEE Tran. VLSI, Vol. 18, No. 3, pp. 378-391, Mar. 2010.

[37] T. Lehtonen, D. Wolpert, P. Liljeberg, J. Plosila and P. Ampadu, "Self-adaptive system for addressing permanent errors in on-chip interconnects," IEEE Tran. VLSI, Vol. 18, No. 4, pp. 527-540, Apr. 2010.

[38]D. Chen, J. Cong, Y. Fan and L. Wan, "LOPASS: A low-power architectural synthesis system for FPGAs with interconnect estimation and optimization,” IEEE Tran. VLSI, Vol. 18, No. 4, pp. 564-577, Apr. 2010.

[39] L. P. Carloni, A. B. Kahng, S. V. Muddu, A. Pinto, K. Samadi, P. Sharma, “Accurate predictive interconnect modeling for system-level design," IEEE Tran. VLSI, Vol. 18, No. 4, pp. 679-684, Apr. 2010.

[40]R. Achar, “Advanced modeling and simulation methodologies for signal integrity analysis”, International Conference on Recent Advances in Microwave Theory and Applications, 2008 (MICROWAVE 2008), pp. 279 - 279, Jaipur, India, 21-24 Nov. 2008.

[41]Agilent EEsof EDA, "Overview: Electromagnetic Design System (EMDS)," (Sep. 2008) [Online]. Available: http://www.agilent.com/find/eesof-emds

[42] Ansoft corporation, "Simulation software: High-performance signal and power integrity," Internal Report, 2006.

[43] ANSYS, "Unparalleled advancements in signal- and power-integrity, electromagnetic compatibility testing," (Jun. 2009) [Online]. Available: http://investors.ansys.com/ 
[44] North East Systems Associates (NESA), "RJ45 interconnect signal integrity," (2010 CST Computer Simulation Technology AG.) [Online]. Available: http://www.cst.com/Content/Applications/Article/Article.aspx?id=243

[45] J. A. Davis and J. D. Meindl, "Compact distributed RLC interconnect models-Part I: Single line transient, time delay and overshoot expressions," IEEE Trans. Electron Devices, Vol. 47, No. 11, Nov.2000, pp. 2068-2077R. M. Corless,

[46] G. H. Gonnet, D. E. G. Hare, D. J. Jeffrey and D. E. Knuth, “On the Lambert W function,” Advances in Computational Mathematics, Vol. 5, 1996, pp. 329-359.

[47] D. J. Jeffrey, D. E. G. Hare and R. M. Corless, "Unwinding the branches of the Lambert W function," Math. Scientist, Vol. 21, 1996, pp. 1-7. 